\title{
Europe's next mission to the Sun
}

\author{
As the Solar Orbiter spacecraft is scheduled for launch this month, European Space Agency (ESA) and NASA \\ Project Scientists provide an overview of this major ESA-NASA mission to the Sun.
}

M ore than four centuries have passed since the first observation of sunspots with a telescope, and more than six decades since the discovery of solar wind. Yet the fundamental mysteries of our star and of its extended atmosphere remain unsolved. Understanding the connections and the coupling between the Sun and the heliosphere is the main goal of Solar Orbiter, a space mission of international collaboration between the European Space Agency (ESA) and NASA. Scheduled to launch in February 2020, the Solar Orbiter spacecraft will be used to study the Sun and the inner heliosphere; it will use a unique combination of six remote-sensing instruments to observe the Sun and solar corona, and four in-situ instruments to measure the solar wind around the spacecraft. Together, the ten Solar Orbiter instruments will provide a complete description of the plasma making up the solar wind - its origin, transport and composition - vastly improving on the Helios missions ${ }^{1}$, launched in 1974 and 1976, and complementing the new measurements of NASA's Parker Solar Probe mission ${ }^{2}$.

Solar Orbiter will reach a minimum perihelion of 0.28 au after a series of Venus and Earth gravity assists, which will also raise the inclination of the orbital plane to more than $33^{\circ}$ from the ecliptic plane, allowing a first-ever look at the solar poles. The design of the mission has been driven by the need to answer the following four interrelated top-level scientific questions ${ }^{3}$ : (1) What drives solar wind, and where does the coronal magnetic field originate? (2) How do solar transients drive heliospheric variability? (3) How do solar eruptions produce the energetic particle radiation that fills the heliosphere? (4) How does the solar dynamo work and drive connections between the Sun and the heliosphere?

To answer these challenging questions, it is essential to make in-situ measurements of the solar wind plasma, fields, waves and energetic particles close enough to the Sun that they are still relatively pristine and have not had their properties modified by subsequent transport and propagation processes. Solar Orbiter will measure the solar wind properties and - at the same time - use its comprehensive telescopes package to try and identify the sources of the plasma detected as it leaves the Sun (Fig. 1). The in-situ instruments

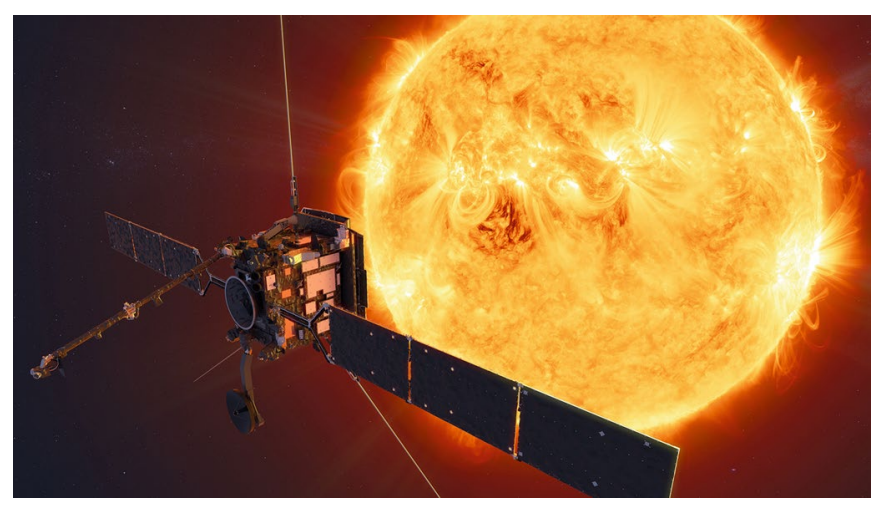

Fig. 1 | Artist's impression of Solar Orbiter. Studying the Sun up close. Credit: ESA/ATG Medialab.

comprise a solar wind analyser (SWA; for measuring the properties of electrons, protons and heavy ions), a magnetometer (MAG), a radio and plasma waves (RPW) experiment and the energetic particle detector (EPD; for measuring higher-energy charged particles) suite. The remote-sensing payload consists of an extreme-ultraviolet full-Sun and high-resolution imager (EUI), a coronagraph (Metis), a polarimetric and helioseismic imager (PHI), a heliospheric imager (SoloHI), an extreme-UV spectral imager (SPICE) and an X-ray telescope and spectrometer (STIX).

Solar Orbiter is the first medium-class mission of ESA's Cosmic Vision programme, developed by scientists and engineers from almost all European countries, and implemented together with NASA. It builds on the success of previous collaboration between ESA and NASA on two major solar missions: SOHO (launched in 1995), a mainly remote-sensing mission; and Ulysses (1990-2009), an in-situ probe that was the first spacecraft to leave the ecliptic. Both have revolutionized the way we think of the Sun and the heliosphere. Solar Orbiter is the conceptual combination of these two missions - an out-of-ecliptic in-situ exploratory probe bringing state-of-the-art telescopes closer to the Sun than ever before. In addition, joint observations with NASA's Parker Solar Probe, which travels so close to the Sun that it could not carry telescopes to observe the solar disk, will deliver new and potentially disruptive results.

The spacecraft is scheduled to launch on 7 February 2020 on an Atlas V 411 launcher from Cape Canaveral. After a short commissioning phase, the in-situ payload will be fully operational as of mid-May, right before Solar Orbiter's first perihelion at 0.51 au in June 2020. The nominal phase, with all 10 instruments operating, will start in November 2021 and run until the end of 2025, with possible extensions up to 2030. Following the open-data philosophy of most solar missions, all science data will be publicly available three months after reception of the data on the ground, a period needed for calibration and checking. The international solar and heliophysics community is now ready for an exciting and busy decade, which will certainly transform our knowledge of the Sun.

\section{Müller (D)1, I. Zouganelis (D)2*, \\ O. C. St. Cyr ${ }^{3}$, H. R. Gilbert ${ }^{3}$ and T. Nieves-Chinchilla ${ }^{3}$}

${ }^{1}$ Science Division, Science and Operations Department, Science Directorate, European Space Agency, ESTEC, Noordwijk, The Netherlands. ${ }^{2}$ Science Division, Science and Operations Department, Science Directorate, European Space Agency, ESAC, Madrid, Spain. ${ }^{3}$ NASA Goddard Space Flight Center, Greenbelt, MD, USA. *e-mail:yannis.zouganelis@esa.int

Published online: 3 February 2020 https://doi.org/10.1038/s41550-020-1015-5

\footnotetext{
References

1. Schwenn, R. \& Marsch, E. (eds) in Physics and Chemistry in Space: Physics of the Inner Heliosphere Vol. 20 (Springer, 1990).

2. Fox, N. J. et al. Space Sci. Rev. 204, 7-48 (2016).

3. Müller, D., Marsden, R. G., St. Cyr, O. C. \& Gilbert, H. R. Sol. Phys. 285, 25-70 (2013).
} 